\title{
Novel Route for Synthesis of Antihypertensive Activity of Tetrazole Analogues as a Carbamate and Urea Derivatives
}

Ramakrishna Vellalacheruvu ${ }^{1,2^{\star}}$, Sai Leela $\mathbf{R}^{3}$ and Ravindranath $\mathbf{L K}^{\mathbf{4}}$

${ }^{1}$ Department of Chemistry, SK University, Anantapur, Andhra Pradesh, India

${ }^{2}$ GVK Bio Science Pvt. Ltd., IDA Nacharam, Secunderabad, Telangana, India

${ }^{3}$ College of Pharmacy, SK University, Anantapur, Andhra Pradesh, India

${ }^{4}$ Chemistry Department, SK University, Andhra Pradesh, India

\begin{abstract}
The Novel route was developed for synthesis of high potential tetrazole carbamate and urea derivatives by using conventional methods. (trifluoromethyl)phenyl)quinoline-5-carboxamide (3) was converted into chloroamidine derivative by using $\mathrm{POCl}_{3}$ and DMF (cat), then treated with sodium azide by [3+2] cycloaddition to give 8-(benzyloxy)-5-(1-(4-(trifluoromethyl) phenyl)-1H-tetrazol-5-yl)quinoline (5). The tetrazolidine compound was debenzylated, then Alkylation with Ethyl Bromo acetate and converted to acid (8) by hydrolysis with $\mathrm{LiOH}$. The acid was converted to acid azide by using DPPA, and then Treated with Alcohols and Amine to give substituted Carbamates and urea derivatives by using Curtius rearrangement.
\end{abstract}

Keywords: Tetrazolidine; Diphenyl phosphoryl azide; [3+2] Cycloaddition reaction; Curties re-arrangement; Combi- flash chromatography

\section{Objective of this Research}

Present work is corresponding to develop novel synthetic route for preparation of the quinoline attached tetrazolidine urea and $\mathrm{N}$-carbamate derivatives and characterized by IR and ${ }^{1} \mathrm{H}$ NMR.

\section{Introduction}

Tetrazole analogues have a potential pharmacological activity in medicinal chemistry division. Several Active pharmaceutical drug intermediates (API) of tetrazole derivatives played their role in pharmaceutical and agrochemical region. These compounds act as multidimensional biological active drug candidates such as inhibition of Angiotensin $\left(\mathrm{AT}_{1}\right)$, Angiotensin $\left(\mathrm{AT}_{2}\right)$ receptor (Hypertension) [1], antifungal and antibacterial [2], corrosion inhibitor [3], antiinflammatory [4], anticancer [5,7], antioxidant [6], antifungal, analgesic [8], Antiviral [9], protein arginine deiminase inhibitor [10], antimicrobial [11,12], Anti allergic, dual Selective serotonin and Nor epinephrine Reuptake inhibitors (SSNRIs) and HIV inhibitors. When Drug model designing, tetrazole nucleolus consider as a co-sister of carboxylic acid and amide derivatives. The pka value of tetrazole is correlated with amide and acid functional groups. The introduction of tetrazole ring in drug substrate prominently increases their bioavailability and prolonging biological action and also avoid acute toxicity of drug. In Analogue-based drug discovery (ABDD), introduce tetrazole nucleolus as an important descriptor. Several research works is progress based on synthesis of tetrazole amino acid analogues, and nucleotide and nucleoside analogues instead of acid and amide functional group.

Many Tetrazole analogs were available for treatment of hypertension, such as Losartan potassium, Valsartan, Irbesartan, Candesartan and Olmesartan medoxonil. These drugs played a vital role to inhibit Angiotensin converting enzyme. They block $\mathrm{AT}_{1}$ and $\mathrm{AT}_{2}$ receptors which is located in kidney, heart, vascular smooth muscle cells, brain, and adrenal glands. The renin angiotensin system (RAS) is a powerful regulator of Blood pressure. These drugs block renin-angiotensin system (RAS, enzyme) which is secretly produced in Kidney. Such few anti-hypertension drugs were described in Figure 1.

Tetrazole ring was widely used as a metabolically stable bioisostere for carboxy and amide functional groups in molecular design and synthesis of modified amino acids. Such analogs are described in Figure 2.

The generations of Cephalosporin Antibiotics played a vital role in diagnosis process. Huge research work has been done for development of these drug analogs shown in Figure 3.

Dave et al. synthesized 7,9-Disubstituted-7H-tetrazolo[1,5-c] pyrrolo $[3,2-e]$ pyrimidines and evaluated their biological activity. Below compound exhibited better activity than ampicillin against all tested culture.

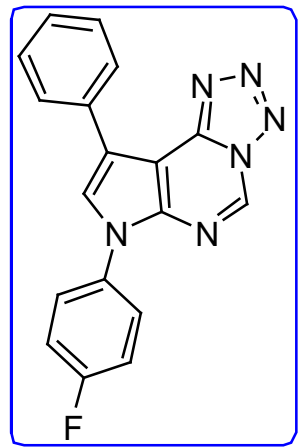

Subramanian et al. developed a novel route for terazole analgous of $\mathrm{Cl}$-Amidines and F- Amidines.

*Corresponding author: Ramakrishna Vellalacheruvu, Department of Chemistry SK University, Anantapur, Andhra Pradesh, India, Tel: 9493268448; E-mail: vellalacheruv@gmail.com

Received August 21, 2017; Accepted August 28, 2017; Published August 31 2017

Citation: Ramakrishna V, Sai Leela R, Ravindranath LK (2017) Novel Route for Synthesis of Antihypertensive Activity of Tetrazole Analogues as a Carbamate and Urea Derivatives. Med Chem (Los Angeles) 7: 239-246. doi: 10.4172/21610444.1000463

Copyright: ( 2017 Ramakrishna V, et al. This is an open-access article distributed under the terms of the Creative Commons Attribution License, which permits unrestricted use, distribution, and reproduction in any medium, provided the original author and source are credited. 
<smiles>CCCCc1nc(Cl)c(CO)n1Cc1ccc(-c2ccccc2-c2nnnn2-c2cnccn2)cc1</smiles>

Losartan Pottasjum<smiles>CCCCC(=O)N(Cc1ccc(-c2ccccc2-c2nnn[nH]2)cc1)C(C)C(C)C</smiles>

valsartan<smiles>CCCCC1=NC2(CCCC2)C(=O)N1Cc1ccc(-c2ccccc2-c2nnn[nH]2)cc1</smiles>

Irbesartan<smiles>CCCc1nc(C(C)(C)O)c(C(=O)OCc2oc(=O)oc2C)n1Cc1ccc(-c2ccccc2-c2nnn[nH]2)cc1</smiles><smiles>CCCc1nc(C(C)(C)O)c(C(=O)OCc2oc(=O)oc2C)n1Cc1ccc(-c2ccccc2-c2nnn[nH]2)cc1</smiles>

Figure 1: List of ACE inhibitors as an anti-hypertensive drugs.<smiles>O=C(O)C1C[C@@H](c2nnn[nH]2)CN1</smiles><smiles>NC(Cc1nnn[nH]1)C(=O)O</smiles><smiles>O=C(O)[C@H]1CCN[C@H](Cc2nn[nH]n2)C1</smiles>

Figure 2: Metabolically bioisostere for carboxy and amide functional groups.

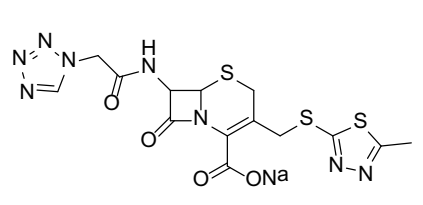

Cefazolin<smiles>Cc1nnc(SCC2=C(C(=O)O)N3C(=O)C(NC(=O)Cn4cnnn4)CSC23)s1</smiles>

Ceftezole<smiles>Cn1nnnc1SCC1=C(C(=O)O)N2C(=O)C(NC(=O)C(OC=O)c3ccccc3)C2SC1</smiles>

Cefamandole

Figure 3: Generation of Cephalosporin Antibiotics.

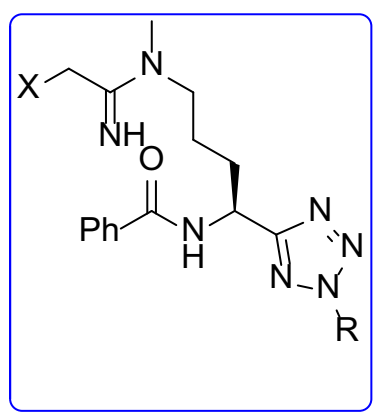

As a part of our research work, we synthesized a high potential tetrazolidine analogous were synthesized as a urea and carbamate derivatives using conventional methods of cycloaddition reactions and curtius rearrangement reactions. Present method we avoid toxic and hazard reagents during synthesis of urea and carbamate derivatives.

\section{Materials and Methods}

All Amines and alcohols and Reagents collected from commercial sources (Aldrich, Alfa Aesar). THF and Toluene were thoroughly dried using sodum metal and benzophenone before conduct reaction. DMF was dried using $\mathrm{CaH}$. Alcohols (EtOH, $\mathrm{MeOH}$ ) were super dried using Grignard method $\left(\mathrm{Mg}, \mathrm{I}_{2}\right)$. The Curtius rearrangement reactions were conducted in sealed tube. These derivatives were characterized 
by using Analytical methods like IR, NMR (400 m Hz, Bruker). The melting points were recorded using on a WRS-1A Digital Melting Point Apparatus without correction. Infrared spectra were taken using an AVATAR 370 FT-IR spectrometer. ${ }^{1} \mathrm{HNMR},{ }^{13} \mathrm{CNMR}$ spectra were recorded with a Bruker spectrometer operating at $400 \mathrm{MHz}$ used as a trimethylsilane as a reference and values were recorded in $\mathrm{ppm}$. The progress of reaction was monitored using TLC system and $I_{2}$ spray and $\mathrm{KMnO}_{4}$ TLC strain. The crude compounds were purified using column chromatography (100-200 mesh silica) and Combi-flash chromatography. The hydrogenation process was carried out using parr shaker.

\section{Experimental Methods}

In this research work, we prepared below compounds and mentioned in step wise manner. The detailed scheme was given in Scheme 1. The Reaction mechanism for step 4 was mentioned in Figure 4.

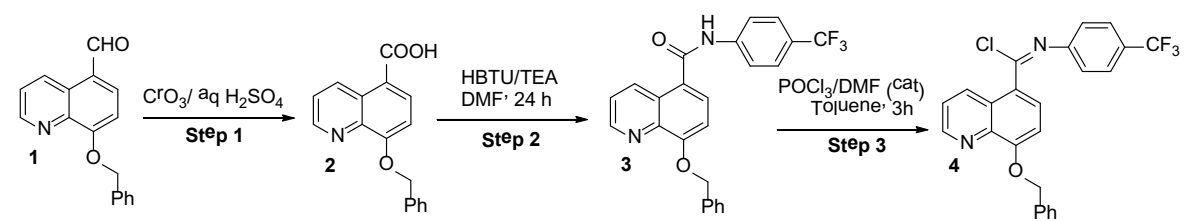
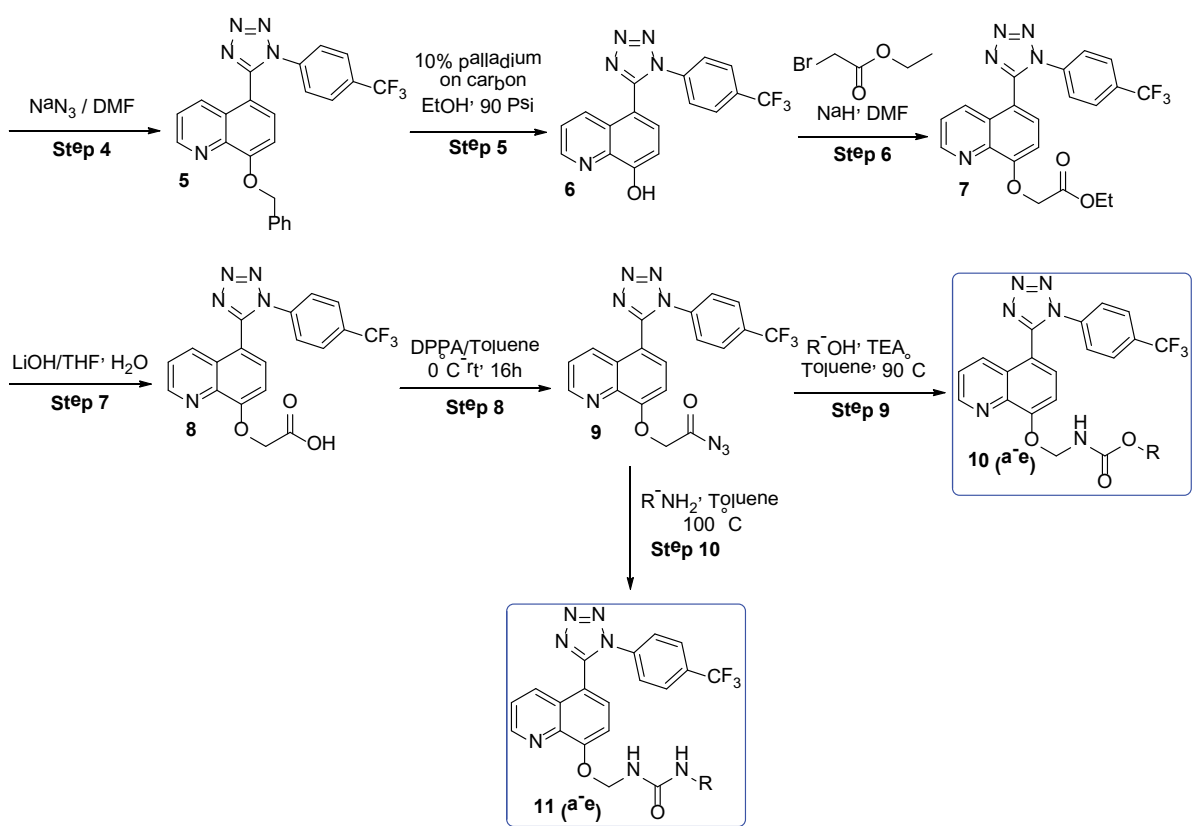

Scheme 1: The detailed scheme of prepared compounds.

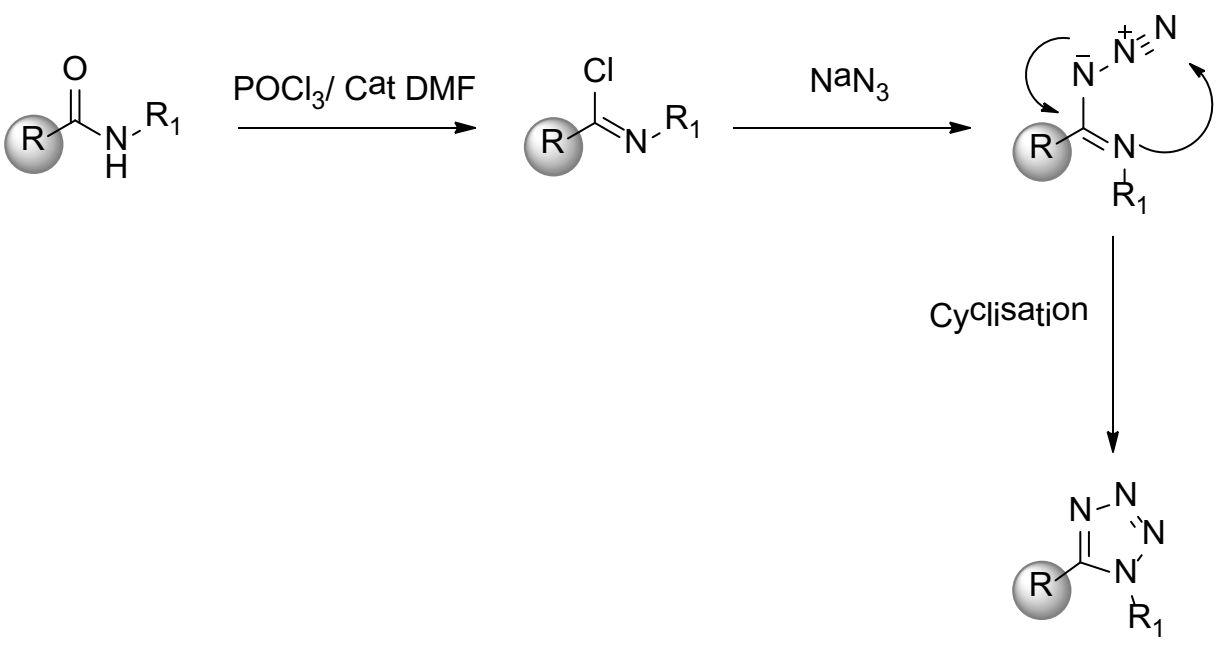

Figure 4: The detailed reaction mechanism for step 4. 
- Step-1: 8-(benzyloxy)quinoline-5-carboxylic acid (2).

- Step-2: 8-(benzyloxy)-N-(4-(trifluoromethyl)phenyl)quinoline5 -carboxamide (3)

- Step-3: (E)-8-(benzyloxy)-N-(4-(trifluoromethyl)phenyl) quinoline-5-carbimidoyl chloride (4).

- Step-4: 8-(benzyloxy)-5-(1-(4-(trifluoromethyl)phenyl)-1Htetrazol-5-yl)quinoline (5).

- Step-5: 5-(1-(4-(trifluoromethyl)phenyl)-1H-tetrazol-5-yl) quinolin-8-ol (6).

- Step-6: Ethyl 2-((5-(1-(4-(trifluoromethyl)phenyl)-1H-tetrazol5-yl)quinolin-8-yl)oxy)acetate (7).

- Step 7: 2-((5-(1-(4-(trifluoromethyl)phenyl)-1H-tetrazol-5-yl) quinolin-8-yl)oxy)acetic acid (8).

- Step 8: 2-((5-(1-(4-(trifluoromethyl)phenyl)-1H-tetrazol-5-yl) quinolin-8-yl)oxy)acetyl azide (9).

- Step 9: Substituted (((5-(1-(4-(trifluoromethyl)phenyl)-1Htetrazol-5-yl)quinolin-8-yl)oxy)methyl)carbamate (10a-e).

- Step 10: substituted-3-(((5-(1-(4-(trifluoromethyl)phenyl)-1Htetrazol-5-yl)quinolin-8-yl)oxy)methyl)urea (11a-e).

\section{Reaction conditions}

Step 1: $\mathrm{CrO}_{3} / \mathrm{H}_{2} \mathrm{SO}_{4}, 0^{\circ} \mathrm{C}, 3 \mathrm{~h}$, Step 2: HBTU /DIPEA (3 eq), DMF Rt, 16 h Step 3: $\mathrm{POCl}_{3} / \mathrm{DMF}$ (cat), $0^{\circ} \mathrm{C}-50^{\circ} \mathrm{C}, 3 \mathrm{~h}$ Step 4: $\mathrm{NaN}_{3} / \mathrm{DMF}$ $80^{\circ} \mathrm{C}$, 4 h Step 5: $10 \%$ Palladium on Carbon, $\mathrm{H}_{2} / 90$ psi Step 6: Ethyl Bromo Acetate, $\mathrm{NaH}, 0^{\circ} \mathrm{C}-\mathrm{RT}, 6$ h Step 7: LiOH, THF/H $\mathrm{H}_{2} \mathrm{O}(1: 4), 16$ h Step 8 : DPPA / Toluene, $0^{\circ} \mathrm{C}$, 8 h Step 9: R-OH, Toluene, $90^{\circ} \mathrm{C}, 5 \mathrm{~h}$, Sealed tube.

\section{Reaction mechanism}

Step 1: 8-(benzyloxy)quinoline-5-carboxylic acid (2):<smiles>O=C(O)c1ccc(OCc2ccccc2)c2ncccc12</smiles>

Zones Reagent: In a 1 lit 3 neck round bottom flask fitted with mechanical stirrer, $\mathrm{CrO}_{3}(28 \mathrm{~g}, 0.285 \mathrm{~mol})$ was dissolve in water $(50$ $\mathrm{mL}$ ) and cooled to $0^{\circ} \mathrm{C}$ for $10 \mathrm{~min}$. Then added $\mathrm{H}_{2} \mathrm{SO}_{4}(5.5 \mathrm{M}, 130 \mathrm{~mL}$, $0.3 \mathrm{eq})$ drop wise for $30 \mathrm{~min}$ at $-5^{\circ} \mathrm{C}$. The reaction mixture was stirred for $10 \mathrm{~min}$. In Another RBF 8-(benzyloxy) quinoline-5-carbaldehyde $(25 \mathrm{~g}, 0.09 \mathrm{~mol})$ was dissolve in Acetone $(250 \mathrm{~mL})$ and cooled to $0^{\circ} \mathrm{C}$. The above reagent (Zones reagent) was added drop wise for $1 \mathrm{~h}$ and stirred for $2 \mathrm{~h}$. The progress of reaction was monitored by TLC. After completion, Reaction mixture was poured in ice cold water and stirred for $30 \mathrm{~min}$. The reaction mixture was extracted with EtOAc $(3 \times 250$ $\mathrm{mL})$. The reaction mixture was filtered on cellite bed. The organic layer were separated and dried over anhydrous $\mathrm{Na}_{2} \mathrm{SO}_{4}$, filtered and evaporated under vacuum to give black crude product. The crude was purified by column chromatography (100-200 mesh silica, Eluent: Pure EtOAc) isolated 8-(benzyloxy)quinoline-5-carboxylic acid (15 g, Yield: $57 \%)$ as a white solid. M.P. $282-285^{\circ} \mathrm{C}$. IR $\left(\mathrm{KBr}, \mathrm{cm}^{-1}\right): 3400,3010,1710$, 1580, 1440, 770, 655. ${ }^{1} \mathrm{HNMR}\left(d_{6}\right.$-DMSO, $\left.400 \mathrm{mHz}\right): 4.9(\mathrm{~s}, 1 \mathrm{H}), 7.35-$ $7.5(\mathrm{~m}, 5 \mathrm{H}), 7.8(\mathrm{t}, 1 \mathrm{H}), 8.4(\mathrm{~d}, 1 \mathrm{H}), 8.9(\mathrm{~d}, 1 \mathrm{H}), 9.5(\mathrm{~d}, 1 \mathrm{H}), 10.2(\mathrm{brs}, 1 \mathrm{H})$.
Step 2: 8-(benzyloxy)-N-(4-(trifluoromethyl)phenyl)quinoline5-carboxamide (3):<smiles>FC(F)(F)c1ccc(/N=C(\Cl)c2ccc(Oc3ccccc3)c3ncccc23)cc1</smiles>

To a mixture of 8-(benzyloxy)quinoline-5-carboxylic acid (15 g, $0.053 \mathrm{~mol}), 4$-(trifluoromethyl) aniline $(8.6 \mathrm{~g}, 0.053 \mathrm{~mol})$ in DMF (100 $\mathrm{mL}$ ) was added Di-isopropyl ethyl amine (DIPEA) $(25 \mathrm{~mL}, 0.159 \mathrm{~mol})$ and cooled to $0^{\circ} \mathrm{C}$. Then HBTU $(22 \mathrm{~g}, 0.06 \mathrm{~mol})$ was added and stirred at room temperature for $16 \mathrm{~h}$. The progress of reaction was monitored by TLC and iodine strain. After completion, reaction mixture was poured in ice cold water $(300 \mathrm{~mL})$ and extracted with EtOAc $(3 \times 300 \mathrm{~mL})$. The organic layer was separated and washed with brine solution $(100$ $\mathrm{mL}$ ). The organic layer was collected and dried over anhydrous $\mathrm{Na}_{2} \mathrm{SO}_{4}$, filtered and evaporated under vacuum to give crude residue. The crude product was purified by Combi-flash column chromatography (230400 meshsilica, Eluent: $10 \% \mathrm{MeOH}-\mathrm{CHCl}_{3}$ ) isolated 8-(benzyloxy)-N(4-(trifluoromethyl) phenyl) quinoline-5-carboxamide (3) (16 g, yield: $72 \%)$ as a white solid. M.p: $352-355^{\circ} \mathrm{C}$. IR $\left(\mathrm{KBr}, \mathrm{cm}^{-1}\right): 3410,3020,1700$ 1610, 1320, 1420, 780, 685. ${ }^{1} \mathrm{HNMR}\left(d_{6}\right.$-DMSO, $\left.400 \mathrm{mHz}\right): 5.1(\mathrm{~s}, 2 \mathrm{H})$, $7.2(\mathrm{~d}, 1 \mathrm{H}), 7.38-7.5(\mathrm{~m}, 5 \mathrm{H}), 7.6(\mathrm{~d}, 2 \mathrm{H}), 7.63(\mathrm{~d}, 2 \mathrm{H}), 7.83(\mathrm{t}, 1 \mathrm{H}), 8.8$ (br, 2H), $9.5(\mathrm{~d}, 1 \mathrm{H})$.

Step 3: (E)-8-(benzyloxy)-N-(4-(trifluoromethyl)phenyl) quinoline-5-carbimidoyl chloride (4):

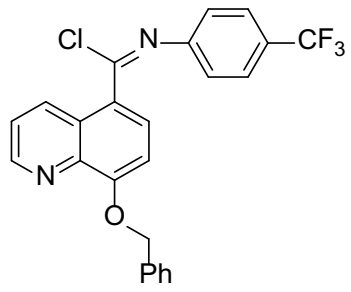

8-(benzyloxy)-N-(4-(trifluoromethyl)phenyl)quinoline-5carboxamide (3) $(15 \mathrm{~g}, 0.035 \mathrm{~mol})$ in $\mathrm{POCl}_{3}(150 \mathrm{~mL})$ was cooled to $0^{\circ} \mathrm{C}$ Then added DMF (cat, $1 \mathrm{~mL}$ ) drop wise and stirred at room temperature for $1 \mathrm{~h}$. The reaction mixture was heated at $60^{\circ} \mathrm{C}$ for $3 \mathrm{~h}$. The progress of reaction was monitored by TLC. After completion, reaction mixture was cooled to room temperature. The $\mathrm{POCl}_{3}$ was evaporated under high vacuum to give crude residue. The residue was co-distilled with $d r y$ toluene $(2 \times 100 \mathrm{ml})$ to give crude product. The crude was carried to next step without further purification. This compound data was not analyzed.

Step 4: 8-(benzyloxy)-5-(1-(4-(trifluoromethyl)phenyl)-1Htetrazol-5-yl)quinoline (5):<smiles>FC(F)(F)c1ccc(-n2nnnc2-c2ccc(OCc3ccccc3)c3ncccc23)cc1</smiles>

(E)-8-(benzyloxy)-N-(4-(trifluoromethyl)phenyl)quinoline-5carbimidoyl chloride (4) $(15 \mathrm{~g}, 0.035 \mathrm{~mol})$ in $d r y \mathrm{DMF}(70 \mathrm{~mL})$ was cooled to $0^{\circ} \mathrm{C}$. Then sodium azide $(3 \mathrm{eq})$ was added portionwise and stirred at room temperature for $1 \mathrm{~h}$. After that, Reaction mixture was heated at $80^{\circ} \mathrm{C}$ for $4 \mathrm{~h}$. The progress of reaction was monitored by TLC. 
After completion, reaction mixture was cooled to $0^{\circ} \mathrm{C}$ and poured in ice cold water $(200 \mathrm{~mL})$ and basified up to $\mathrm{P}^{\mathrm{H}}-8$ with sat aq $\mathrm{NaHCO}_{3}$ sol. The aqueous layer was extracted with EtOAc $(3 \times 100 \mathrm{~mL})$. The organic layer were collected and dried over anhydrous $\mathrm{Na}_{2} \mathrm{SO}_{4}$, filtered and evaporated under vacuum to give crude product. The crude was purified by column chromatography (100-200 mesh silica, Eluent: $50 \%$ EtOAc-Pet Ether) isolated 8-(benzyloxy)-5-(1-(4-(trifluoromethyl) phenyl)-1H-tetrazol-5-yl) quinoline (5) (8 g, Yield : 50\%) as a pale yellow solid. M.p: $280-282^{\circ} \mathrm{C}$. IR $\left(\mathrm{KBr}, \mathrm{cm}^{-1}\right): 3010,1550,1510,820,655$ ${ }^{1} \mathrm{HNMR}\left(d_{6}\right.$-DMSO, $\left.400 \mathrm{mHz}\right): 5.10(\mathrm{~s}, 2 \mathrm{H}), 7.1(\mathrm{~d}, 1 \mathrm{H}), 7.4-7.68(\mathrm{~m}$, $11 \mathrm{H}), 8.4(\mathrm{~d}, 1 \mathrm{H}), 8.78(\mathrm{~d}, 1 \mathrm{H})$.

Step 5: 5-(1-(4-(trifluoromethyl)phenyl)-1H-tetrazol-5-yl) quinolin-8-ol (5):<smiles>Oc1ccc(-c2nnnn2-c2ccc(C(F)(F)F)cc2)c2cccnc12</smiles>

8-(benzyloxy)-5-(1-(4-(trifluoromethyl)phenyl)-1H-tetrazol-5yl)quinoline (5) $(8 \mathrm{~g}, 0.012 \mathrm{~mol})$ in $\mathrm{MeOH}(100 \mathrm{~mL})$ was added $10 \%$ palladium on carbon and TFA $(10 \mathrm{~mL})$ and hydrogenated at 80 Psi using parr shaker for $5 \mathrm{~h}$ at room temperature. The progress of reaction was monitored by TLC. After completion, Reaction mixture was filtered on cellite bed and washed with $\mathrm{MeOH}(2 \times 50 \mathrm{~mL})$. The organic layer was collected and evaporated under vacuum to give crude residue. The residue was purified by combi-flash column chromatography isolated 5-(1-(4-(trifluoromethyl) phenyl)-1H-tetrazol-5-yl) quinolin-8-ol (6) (5.5 g, Yield: $88 \%)$. as a white solid. M.p:310-315 ${ }^{\circ} \mathrm{C}$. IR $\left(\mathrm{KBr}, \mathrm{cm}^{-1}\right)$ : $3300,3040,1580,1425,1150,760,691,{ }^{1} \operatorname{HNMR}\left(d_{6}\right.$-DMSO, $\left.400 \mathrm{mHz}\right)$ : $7.2(\mathrm{~d}, 1 \mathrm{H}), 7.5-7.62(\mathrm{~m}, 5 \mathrm{H}), 7.8(\mathrm{~d}, 1 \mathrm{H}), 8.4(\mathrm{~d}, 1 \mathrm{H}), 8.88(\mathrm{~d}, 1 \mathrm{H}) .10 .1$ (brs, $1 \mathrm{H}$ ).

Step 6: Ethyl 2-((5-(1-(4-(trifluoromethyl)phenyl)-1H-tetrazol5 -yl)quinolin-8-yl)oxy)acetate (7):<smiles>CCOC(=O)COc1ccc(-c2nnnn2-c2ccc(C(F)(F)F)cc2)c2cccnc12</smiles>

To a solution 5-(1-(4-(trifluoromethyl)phenyl)-1H-tetrazol-5yl)quinolin-8-ol (6) (5 g, $0.014 \mathrm{~mol})$, in DMF (50 mL) was cooled to $0^{\circ} \mathrm{C}$. Then added sodium hydride $(1.7 \mathrm{~g}, 0.042 \mathrm{~mol}, 3 \mathrm{eq})$ portion wise and stirred for $30 \mathrm{~min}$. To that Ethyl bromo acetate $(1.71 \mathrm{~mL}, 0.0154$ mol) was added drop wise and stirred for $6 \mathrm{~h}$ at room temperature. The progress of reaction was monitored by TLC. After completion, reaction mixture was poured in ice cold water $(100 \mathrm{~mL})$ and basified with aq $\mathrm{Na}_{2} \mathrm{CO}_{3}$ up to $\mathrm{P}_{-}-8$ and extracted with EtOAc $(2 \times 100 \mathrm{~mL})$. The organic layer were separated and washed with brine $(25 \mathrm{~mL})$, and dried over anhydrous $\mathrm{Na}_{2} \mathrm{SO}_{4}$, filtered and evaporated under vacuum to give give crude product. The crude was purified by column chromatography (100-200 mesh silica, Eluent: 60\% EtOAc- Pet ether) isolated ethyl 2-((5-(1-(4-(trifluoromethyl) phenyl)-1H-tetrazol-5-yl)quinolin-8-yl) oxy)acetate ( $4 \mathrm{~g}$, Yield: $64 \%$ ) as a semi brown colour liquid. M.p::255$258^{\circ} \mathrm{C}$. IR $\left(\mathrm{KBr}, \mathrm{cm}^{-1}\right): 3040,1640,1560,1440,1130,780,698,{ }^{1} \mathrm{HNMR}$ $\left(d_{6}-\mathrm{DMSO}, 400 \mathrm{mHz}\right): 1.4(\mathrm{t}, 3 \mathrm{H}), 3.8(\mathrm{q}, 2 \mathrm{H}), 4.8(\mathrm{~s}, 2 \mathrm{H}), 7.23(\mathrm{~d}, 1 \mathrm{H})$, 7.5-7.62 (m, 5H), $7.75(\mathrm{~d}, 1 \mathrm{H}), 8.39(\mathrm{~d}, 1 \mathrm{H}), 8.7(\mathrm{~d}, 1 \mathrm{H})$.
Step 7: 2-((5-(1-(4-(trifluoromethyl)phenyl)-1H-tetrazol-5-yl) quinolin-8-yl)oxy)acetic acid (8):<smiles>CCOC(=O)COc1ccc(-c2nnnn2-c2ccc(C(F)(F)F)cc2)c2cccnc12</smiles>

Ethyl 2-((5-(1-(4-(trifluoromethyl) phenyl)-1H-tetrazol-5-yl) quinolin-8-yl)oxy)acetate (8) (4 g, $0.009 \mathrm{~mol})$ in THF/ $\mathrm{H}_{2} \mathrm{O}(40 \mathrm{~mL} / 10$ $\mathrm{mL}, 4: 1$ ) was added $\mathrm{LiOH}$ (4 eq) and stirred at room temperature for 16 h. The progress of reaction was monitored by TLC. After completion, reaction mixture was poured in ice cold water $(100 \mathrm{~mL})$ and acidified with aq $\mathrm{NaHSO}_{3}(100 \mathrm{~mL})$ up to $\mathrm{P}_{-}-4$ and extracted with EtOAc $(3 \times 100$ $\mathrm{mL}$ ). The organic layer were collected and dried over anhydrous $\mathrm{Na}_{2} \mathrm{SO}_{4}$, filtered and evaporated under vacuum to give crude product. The crude was purified by column chromatography (100-200 mesh silica, Eluent: 80\% EtOAc- Pet ether) isolated 2-((5-(1-(4-(trifluoromethyl) phenyl)1H-tetrazol-5-yl)quinolin-8-yl)oxy)acetic acid (3.5, Yield: 93\%) as a white solid. M.p: $285-288^{\circ} \mathrm{C}$. IR $\left(\mathrm{KBr}, \mathrm{cm}^{-1}\right): 3500,3030,1690,1560$, 1440, 760, 610, ${ }^{1} \mathrm{HNMR}\left(d_{6}\right.$-DMSO, $\left.400 \mathrm{mHz}\right): 4.8$ (s, 2H), 7.3 (d, $\left.1 \mathrm{H}\right)$, 7.5-7.65 (m, 5H), $7.78(\mathrm{~d}, 1 \mathrm{H}), 8.38(\mathrm{~d}, 1 \mathrm{H}), 8.8(\mathrm{~d}, 1 \mathrm{H}), 10.4$ (brs, $1 \mathrm{H})$.

Step 8: 2-((5-(1-(4-(trifluoromethyl)phenyl)-1H-tetrazol-5-yl) quinolin-8-yl)oxy)acetyl azide (9):<smiles>N#CCOc1ccc(-c2nnnn2-c2ccc(C(F)(F)F)cc2)c2cccnc12</smiles>

To a solution of 2-((5-(1-(4-(trifluoromethyl)phenyl)-1H-tetrazol5-yl)quinolin-8-yl)oxy) acetic acid (3.5 g, $0.008 \mathrm{~mol})$ in toluene (35 $\mathrm{mL})$ was added DPPA $(2.25 \mathrm{~mL}, 0.014 \mathrm{~mol})$ at $0^{\circ} \mathrm{C}$ and stirred at room temperature for $8 \mathrm{~h}$. The progress of reaction was monitored by TLC. After completion, reaction mixture was evaporated under vacuum to give crude residue. The crude residue was co-distilled with Toluene (2 $\times 30 \mathrm{~mL})$ isolated 2-((5-(1-(4-(trifluoromethyl) phenyl)-1H-tetrazol-5yl)quinolin-8-yl)oxy)acetyl azide (4.5 g) as a liquid. The crude residue was carried to next step without further purification.

Step 9: Substituted ((5-(1-(4-(trifluoromethyl)phenyl)-1Htetrazol-5-yl)quinolin-8-yl)oxy)methyl)carbamate (10):<smiles>[R]C(=O)NCOc1ccc(-c2nnnn2-c2ccc(C(F)(F)F)cc2)c2cccnc12</smiles>

To a solution of 2-((5-(1-(4-(trifluoromethyl)phenyl)-1H-tetrazol5-yl)quinolin-8-yl)oxy)acetyl azide (9) $(250 \mathrm{mg}, 0.568 \mathrm{mmol})$ in Dry toluene $(10 \mathrm{~mL})$ was added TEA $(1.7 \mathrm{mmol}, 3 \mathrm{eq})$, Alcohol $(1.1 \mathrm{eq})$ and heated at $100^{\circ} \mathrm{C}$ for $5 \mathrm{~h}$ in sealed tube $(50 \mathrm{~mL})$. The progress of reaction 
Citation: Ramakrishna V, Sai Leela R, Ravindranath LK (2017) Novel Route for Synthesis of Antihypertensive Activity of Tetrazole Analogues as a Carbamate and Urea Derivatives. Med Chem (Los Angeles) 7: 239-246. doi: 10.4172/2161-0444.1000463

was monitored by TLC. After completion, reaction was evaporated under vacuum to give crude product. The crude was purified by Combi-flash column chromatography (100-200 mesh silica, Eluent: $70 \%$ EtOAc-Pet ether), isolated substituted (((5-(1-(4-(trifluoromethyl) phenyl)-1H-tetrazol-5-yl)quino lin-8-yl)oxy)methyl)carbamate 10 (ae). The list of carbonate derivatives was given in Table 1 .

Methyl(((5-(1-(4-(trifluoromethyl)phenyl)-1H-tetrazol-5-yl) quinolin-8-yl)oxy)methyl) carbamate (10 a):<smiles>COC(=O)NCOc1ccc(-c2nnnn2-c2ccc(C(F)(F)F)cc2)c2ccccc12</smiles>

M.p: $280-283^{\circ} \mathrm{C} . \mathrm{IR}\left(\mathrm{KBr}, \mathrm{cm}^{-1}\right): 3450,3030,1645,1350,780,645$, ${ }^{1} \mathrm{HNMR}\left(d_{6}\right.$-DMSO, $\left.400 \mathrm{mHz}\right): 3.5(\mathrm{~s}, 2 \mathrm{H}), 5.5(\mathrm{~s}, 2 \mathrm{H}), 7.2(\mathrm{~d}, 1 \mathrm{H}), 7.5-$ $7.64(\mathrm{~m}, 5 \mathrm{H}), 8.1(\mathrm{~m}, 3 \mathrm{H}), 8.4(\mathrm{~d}, 1 \mathrm{H}), 8.8(\mathrm{~d}, 1 \mathrm{H}) .{ }^{13} \mathrm{CNMR}\left(d_{6}\right.$-DMSO, $400 \mathrm{mHz}): 51,75,108,122,123,124.5,125,131,132,135,139,149,155$, 157, 163.

Ethyl ((5-(1-(4-(trifluoromethyl)phenyl)-1H-tetrazol-5-yl) quinolin-8-yl)oxy)methyl) carbamate $(10 \mathrm{~b})$ :<smiles>CCOC(=O)NCOc1ccc(-c2nnnn2-c2ccc(C(F)(F)F)cc2)c2cccnc12</smiles>

M.p: $290-293^{\circ} \mathrm{C} . \mathrm{IR}\left(\mathrm{KBr}, \mathrm{cm}^{-1}\right): 3450,3035,1650,1350,785,650$, ${ }^{1} \mathrm{HNMR}\left(d_{6}\right.$-DMSO, $\left.400 \mathrm{mHz}\right): 1.25(\mathrm{t}, 3 \mathrm{H}), 4.2(\mathrm{q}, 2 \mathrm{H}), 5.4(\mathrm{~s}, 2 \mathrm{H})$, $7.2(\mathrm{~d}, 1 \mathrm{H})$ 7.55-7.63 (m, 5H), $7.9(\mathrm{~m}, 2 \mathrm{H}), 8.38(\mathrm{~d}, 1 \mathrm{H}), 8.85(\mathrm{~d}, 1 \mathrm{H})$. ${ }^{13}$ CNMR (d - DMSO, $400 \mathrm{mHz}$ ): 15, 62, 77, 107.5, 122, 123, 124, 125, 131, 132, 135, 140, 150, 155, 156, 164.

Tert-butyl(((5-(1-(4-(trifluoromethyl)phenyl)-1H-tetrazol-5-yl) quinolin-8-yl)oxy)methyl) carbamate $(10 \mathrm{c})$ :<smiles>CC(C)(C)OC(=O)NCOc1ccc(-c2nnnn2-c2ccc(C(F)(F)F)cc2)c2cccnc12</smiles>

M.P.: $250-253^{\circ} \mathrm{C}$, IR $\left(\mathrm{KBr}, \mathrm{cm}^{-1}\right): .3470,3040,1670,1380,770,640$. ${ }^{1} \mathrm{HNMR}\left(d_{6}\right.$-DMSO, $\left.400 \mathrm{mHz}\right): 1.5(\mathrm{~s}, 9 \mathrm{H}), 5.5(\mathrm{~s}, 2 \mathrm{H}), 7.18(\mathrm{~d}, 1 \mathrm{H})$

\begin{tabular}{|c|c|c|c|}
\hline Compounds (10a-f) & $\mathbf{R}$ & Reaction Time & Yield \\
\hline $\mathbf{1 0} \mathbf{a}$ & $\mathrm{Me}$ & $5 \mathrm{~h}$ & $40 \%$ \\
\hline $\mathbf{1 0} \mathbf{b}$ & $\mathrm{Et}$ & $8 \mathrm{~h}$ & $55 \%$ \\
\hline $\mathbf{1 0} \mathbf{c}$ & $\mathrm{tBu}$ & $16 \mathrm{~h}$ & $60 \%$ \\
\hline $\mathbf{1 0} \mathbf{d}$ & $\mathrm{Benzyl}$ & $10 \mathrm{~h}$ & $50 \%$ \\
\hline $\mathbf{1 0} \mathbf{e}$ & $\mathrm{MeO}-\mathrm{C}_{6} \mathrm{H}_{4}-\mathrm{CH}_{2}$ & $6 \mathrm{~h}$ & $65 \%$ \\
\hline
\end{tabular}

Table 1: Summary data for $\mathrm{N}$-carbamate derivatives.
7.55-7.63 (m, 5H), $7.8(\mathrm{~m}, 2 \mathrm{H}), 8.37(\mathrm{~d}, 1 \mathrm{H}), 8.83(\mathrm{~d}, 1 \mathrm{H}) .{ }^{13} \mathrm{CNMR}\left(d_{6}\right.$ DMSO, $400 \mathrm{mHz}$ ): 30, 76, 79, 107, 121, 122.8, 124.9, 131, 132, 136, 139, $148,155,156,163.8$.

Benzyl (((5-(1-(4-(trifluoromethyl)phenyl)-1H-tetrazol-5-yl) quinolin-8-yl)oxy)methyl) carbamate (10 d):<smiles>O=C(NCOc1ccc(-c2nnnn2-c2ccc(C(F)(F)F)cc2)c2cccnc12)OCc1ccccc1</smiles>

M.p: $300-303^{\circ} \mathrm{C}$. IR $\left(\mathrm{KBr}, \mathrm{cm}^{-1}\right): 3490,3030,1650,1380,780,660$. ${ }^{1} \mathrm{HNMR}\left(d_{6}\right.$-DMSO, $\left.400 \mathrm{mHz}\right): 5.1(\mathrm{~s}, 2 \mathrm{H}), 5.6(\mathrm{~s}, 2 \mathrm{H}), 7.2(\mathrm{~d}, 1 \mathrm{H}) 7.55-$ $7.63(\mathrm{~m}, 10 \mathrm{H}), 7.85(\mathrm{~m}, 2 \mathrm{H}), 8.39(\mathrm{~d}, 1 \mathrm{H}), 8.85(\mathrm{~d}, 1 \mathrm{H}) .{ }^{13} \mathrm{CNMR}\left(d_{6}-\right.$ DMSO, $400 \mathrm{mHz}): 68,78,108,122,123,124,124.5,125.3,127,127.6$, 129, 131, 132, 135.2, 136, 139, 148, 155, 156, 163.

4-methoxybenzyl ((5-(1-(4-(trifluoromethyl)phenyl)-1Htetrazol-5-yl)quinolin-8-yl)oxy)methyl)carbamate (10 e)

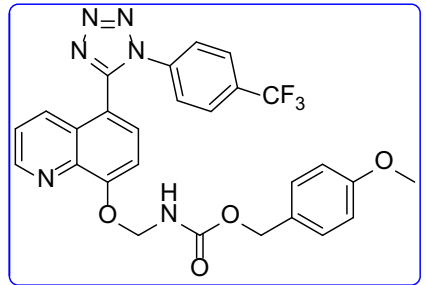

M.P.320-323 ${ }^{\circ} \mathrm{C}$, IR $\left(\mathrm{KBr}, \mathrm{cm}^{-1}\right): 3460,3050,1640,1370,785,665$. ${ }^{1} \mathrm{HNMR}\left(d_{6}\right.$-DMSO, $\left.400 \mathrm{mHz}\right): 3.83(\mathrm{~s}, 3 \mathrm{H}), 4.9(\mathrm{~s}, 2 \mathrm{H}), 5.65(\mathrm{~s}, 2 \mathrm{H})$, 6.9-7.1 (m, 5H), 7.55-7.62 (m, $5 \mathrm{H}), 7.85(\mathrm{~m}, 2 \mathrm{H}), 8.38(\mathrm{~d}, 1 \mathrm{H}), 8.87$ $(\mathrm{d}, 1 \mathrm{H}) \cdot{ }^{13} \mathrm{CNMR}\left(d_{6}\right.$-DMSO, $\left.400 \mathrm{mHz}\right): 56,67,76,108,115,122,123$, $123.8,124,124.1,125.3,128,129,130,131.9,132,135.2,139,149,154$, $156,158,164$

Step 10: 3-((5-(1-(4-(trifluoromethyl)phenyl)-1H-tetrazol-5-yl) quinolin-8-yl)oxy)methyl) urea derivative (11):<smiles>[R]C(=O)NCOc1ccc(-c2nnnn2-c2ccc(C(F)(F)F)cc2)c2cccnc12</smiles>

To a solution of 2-((5-(1-(4-(trifluoromethyl)phenyl)-1H-tetrazol5-yl)quinolin-8-yl)oxy)acetyl azide (9) (250 mg, $0.568 \mathrm{mmol})$ in Dry toluene $(10 \mathrm{~mL})$ was added Amine $(1.3 \mathrm{eq})$ and heated at $100^{\circ} \mathrm{C}$ for $5 \mathrm{~h}$ in sealed tube $(50 \mathrm{~mL})$. The progress of reaction was monitored by TLC. After completion, reaction was evaporated under vacuum to give crude product. The crude was purified by Combi-flash column chromatography (100-200 mesh silica, Eluent: (5-10\% $\left.\mathrm{MeOH}-\mathrm{CHCl}_{3}\right)$, isolated 1-(((5-(1-(4-(trifluoromethyl)phenyl)-1H-tetrazol-5-yl) quinolin-8-yl)oxy)methyl) urea derivatives 11 (a-e). The list of urea derivatives was mentioned in Table 2. 
Citation: Ramakrishna V, Sai Leela R, Ravindranath LK (2017) Novel Route for Synthesis of Antihypertensive Activity of Tetrazole Analogues as a Carbamate and Urea Derivatives. Med Chem (Los Angeles) 7: 239-246. doi: 10.4172/2161-0444.1000463

\begin{tabular}{|c|c|c|c|c|c|}
\hline Compounds 11(a-e) & 11a & 11b & 11c & 11d \\
\hline $\mathbf{R}$ & 11e & \\
\hline Reaction Time & $60 \%$ & $10 \mathrm{~h}$ & $8 \mathrm{~h}$ & \\
\hline Yield & $40 \%$ & $55 \%$ & $\mathrm{~h}$ & $45 \%$ \\
\hline
\end{tabular}

Table 2: The summary of urea derivatives $11(\mathrm{a}-\mathrm{e})$.

1,1-diethyl-3-((5-(1-(4-(trifluoromethyl)phenyl)-1H-tetrazol-5yl)quinolin-8-yl)oxy)methyl) urea (11 a): IR $\left(\mathrm{KBr}, \mathrm{cm}^{-1}\right)$ :

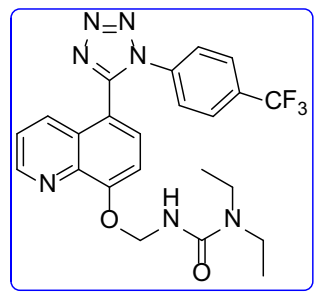

White solid, IR $\left(\mathrm{KBr}, \mathrm{cm}^{-1}\right): 3410,3010,1730,1655,1450,1320,780$, 650,. ${ }^{1} \mathrm{HNMR}\left(d_{6}-\mathrm{DMSO}, 400 \mathrm{mHz}\right): 1.5(\mathrm{t}, 6 \mathrm{H}), 3.8(\mathrm{q}, 4 \mathrm{H}), 5.4(\mathrm{~s}, 2 \mathrm{H})$, $7.15(\mathrm{~d}, 1 \mathrm{H}), 7.5-7.6(\mathrm{~m}, 5 \mathrm{H}), 7.8(\mathrm{~m}, 2 \mathrm{H}), 8.38(\mathrm{~d}, 1 \mathrm{H}), 8.8(\mathrm{~d}, 1 \mathrm{H})$. ${ }^{13} \mathrm{CNMR}\left(d_{6}\right.$-DMSO, $\left.400 \mathrm{mHz}\right): 15,45,78,108,122,123,124,124.5$, $125,131,132,135,139,149,155,157,164$.

11,1-diisopropyl-3-(((5-(1-(4-(trifluoromethyl)phenyl)-1Htetrazol-5-yl)quinolin-8-yl)oxy) methyl)urea (11 b): IR (KBr, $\left.\mathrm{cm}^{-1}\right)$ :<smiles></smiles>

Off white colour solid, IR $\left(\mathrm{KBr}, \mathrm{cm}^{-1}\right): 3430,3020,1720,1645,1440$, 1330,760, 640. ${ }^{1} \mathrm{HNMR}\left(d_{6}-\mathrm{DMSO}, 400 \mathrm{mHz}\right): 1.4(\mathrm{~d}, 12 \mathrm{H}), 3.8(\mathrm{q}$, $2 \mathrm{H}), 5.6(\mathrm{~s}, 2 \mathrm{H}), 7.1(\mathrm{~d}, 1 \mathrm{H}), 7.5-7.6(\mathrm{~m}, 5 \mathrm{H}), 7.8(\mathrm{~m}, 2 \mathrm{H}), 8.4(\mathrm{~d}, 1 \mathrm{H})$, 8.83(d, $1 \mathrm{H}) .{ }^{13} \mathrm{CNMR}\left(d_{6}\right.$-DMSO, $\left.400 \mathrm{mHz}\right): 21,55,77,107,121,123$, 124, 124.5, 131, 132, 133, 135, 139, 148, 154, 158, 165.

1-(tert-butyl)-3-((5-(1-(4-(trifluoromethyl)phenyl)-1H-tetrazol5-yl)quinolin-8-yl)oxy) methyl) urea (11 c): IR $\left(\mathrm{KBr}, \mathrm{cm}^{-1}\right)$ :

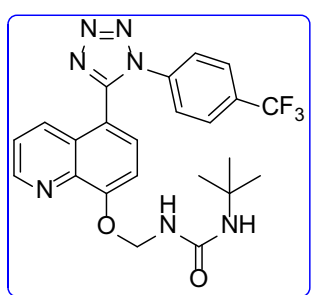

White colour solid, IR $\left(\mathrm{KBr}, \mathrm{cm}^{-1}\right): 3460,3040,1650,1460,1380$, 680, 620. ${ }^{1} \mathrm{HNMR}$ ( $\mathrm{d}_{6}$-DMSO, $\left.400 \mathrm{mHz}\right): 1.5(\mathrm{~s}, 9 \mathrm{H}), 5.68(\mathrm{~s}, 2 \mathrm{H}), 7.15$ $(\mathrm{d}, 1 \mathrm{H}), 7.5-7.6(\mathrm{~m}, 5 \mathrm{H}), 7.8(\mathrm{~m}, 2 \mathrm{H}), 8.1(\mathrm{brs}, 1 \mathrm{H}), 8.43(\mathrm{~d}, 1 \mathrm{H}), 8.84(\mathrm{~d}$, $1 \mathrm{H}) .{ }^{13} \mathrm{CNMR}\left(d_{6}\right.$-DMSO, $\left.400 \mathrm{mHz}\right): 30,58,76,108,122,123,124$, $124.5,131.8,132,133,135,139,146,155,157,164$.

1-Cyclopropyl-3-((5-(1-(4-(trifluoromethyl)phenyl)-1Htetrazol-5-yl)quinolin-8-yl)oxy)methyl)urea (11 d): IR (KBr, $\left.\mathrm{cm}^{-1}\right)$ :<smiles>O=C(NCOc1ccc(-c2nnnn2-c2ccc(C(F)(F)F)cc2)c2ccccc12)NC1CC1</smiles>

Pale yellow colour solid, IR $\left(\mathrm{KBr}, \mathrm{cm}^{-1}\right)$ : 3510, 3430, 3040, 1650, 1460, 1340, 690, 550. ${ }^{1} \mathrm{HNMR}\left(d_{6}-\mathrm{DMSO}, 400 \mathrm{mHz}\right): 0.9(\mathrm{t}, 2 \mathrm{H}), 1.1(\mathrm{t}$, $2 \mathrm{H}), 2.5(\mathrm{~m}, 1 \mathrm{H}), 5.7(\mathrm{~s}, 2 \mathrm{H}), 3.8$ (brs, $1 \mathrm{H}), 7.15(\mathrm{~d}, 1 \mathrm{H}), 7.5-7.6(\mathrm{~m}$, $5 \mathrm{H}), 7.81(\mathrm{~m}, 2 \mathrm{H}), 8.3(\mathrm{brs}, 1 \mathrm{H}), 8.4(\mathrm{~d}, 1 \mathrm{H}), 8.80(\mathrm{~d}, 1 \mathrm{H}) .{ }^{13} \mathrm{CNMR}\left(d_{6}-\right.$ DMSO, $400 \mathrm{mHz}): 8,30,76,108,122,123.1,123.5,124,125.3,130.1$, $132,135,139.1,149,155,157,164$.

1-(4-methoxybenzyl)-3-((5-(1-(4-(trifluoromethyl)phenyl)-1Htetrazol-5-yl)quinolin-8-yl)oxy)methyl)urea (11 e):<smiles></smiles>

White colour solid, IR $\left(\mathrm{KBr}, \mathrm{cm}^{-1}\right)$ : 3540, 3430, 3030, 1640, 1440, 1350, 730. ${ }^{1} \mathrm{HNMR}\left(d_{6}\right.$-DMSO, $\left.400 \mathrm{mHz}\right): 3.6(\mathrm{~s}, 3 \mathrm{H}), 4.2(\mathrm{~s}, 2 \mathrm{H}), 5.7$ (s, 2H), $6.8(\mathrm{~d}, 2 \mathrm{H}), 7.1(\mathrm{~d}, 1 \mathrm{H}), 7.25(\mathrm{~d}, 2 \mathrm{H}), 7.5-7.62(\mathrm{~m}, 5 \mathrm{H}), 7.83(\mathrm{~m}$, $2 \mathrm{H}), 8.25$ (brs, $1 \mathrm{H}), 8.41(\mathrm{~d}, 1 \mathrm{H}), 8.84(\mathrm{~d}, 1 \mathrm{H}) .{ }^{13} \mathrm{CNMR}\left(d_{6}\right.$-DMSO, 400 $\mathrm{mHz}): 45,56,77,107.5,115,121,122,123,124,130,130.5,130.8,132.2$, $135,140,149,155,157,158,163$.

\section{Conclusion}

Present research work, we have developed a novel route for synthesis of high potential pharmacological carbamate and urea derivatives using conventional and scalable route. We avoid by products in curtius rearrangement by using $d r y$ toluene and reactions were executed under argon atmosphere. We avoid hazard reagents like (phosgene and acid chloride methods) for preparation of urea and carbamate derivatives. We employed very easiest method for preparation of urea and carbamate derivatives and avoid side products. We are planning to these derivatives check for biological evolution. The biological evolution details will include next article.

\section{Acknowledgements}

I sincerely thank my guide, Dr. LK Ravindranath and Co-workers, Department of Chemistry, Sri Krishnadevaraya University, Anantapur, Andhra Pradesh, India and GVK Bio Sciences for providing laboratory and analytical facilities.

\section{References}

1. Vangala VB, Hindupur RM, Pati HN (2014) A Review on Synthesis of 
Citation: Ramakrishna V, Sai Leela R, Ravindranath LK (2017) Novel Route for Synthesis of Antihypertensive Activity of Tetrazole Analogues as a Carbamate and Urea Derivatives. Med Chem (Los Angeles) 7: 239-246. doi: 10.4172/2161-0444.1000463

Antihypertensive Sartan Drugs. International Journal of Pharmaceutical Sciences Review and Research 3: 46-56.

2. Maruthamuthu M, Rajam S, Stella $C R$, Bharathi Dileepan AG, Ranjith $R$ (2016) The chemistry and biological significance of imidazole, benzimidazole benzoxazole, tetrazole and quinazolinone nucleus. Journal of Chemical and Pharmaceutical Research 8: 505-526.

3. Rajendran M, Devapiriam D (2015) DFT calculations for corrosion inhibition of copper by tetrazole derivatives. Journal of Chemical and Pharmaceutical Research 7: 763-773.

4. Anu MM, Jayanthi M, Kumar SD, Raja S, Thirunavukkarasu SV (2013) Synthesis, characterization, antibacterial \& anti-inflammatory effects of substituted tetrazole derivatives based on different types of carbazone and benzaldehyde. Synthesis 5: 1982-1990.

5. Bhaskar VH, Mohite PB (2010) Synthesis, characterization and evaluation of anticancer activity of some tetrazole derivatives. Journal of Optoelectronics and Biomedical Materials 2: 249-259.

6. Kale MA, Peharkar MR (2013) Synthesis of some novel tetrazole substituted benzimidazoles and their evaluation as antioxidants. Int J Pharm Bio Sci 4:
675-681.

7. Habibi D, Nabavi H, Nasrollahzadeh M (2013) Silica sulfuric acid as an efficient heterogeneous catalyst for the solvent-free synthesis of 1 -substituted $1 \mathrm{H}-1,2$, 3, 4-tetrazoles. Journal of Chemistry.

8. Wei CX, Bian M, Gong GH (2015) Tetrazolium compounds: synthesis and applications in medicine. Molecules 20: 5528-5553.

9. Darvish F, Khazraee S (2015) FeCl3 Catalyzed One Pot Synthesis of 1-Substituted $1 \mathrm{H}-1,2,3$, 4-Tetrazoles under Solvent-Free Conditions. International Journal of Organic Chemistry 5: 75

10. Subramanian V, Knight JS, Parelkar S, Anguish L, Coonrod SA, et al. (2015) Design, synthesis, and biological evaluation of tetrazole analogs of $\mathrm{Cl}$-amidine as protein arginine deiminase inhibitors. Journal of Medicinal Chemistry 58: 1337-1344.

11. Ostrovskii VA, Trifonov RE, Popova EA (2012) Medicinal chemistry of tetrazoles. Russ Chem Bull 61: 768-780.

12. Mohite PB, Bhaskar VH (2011) Potential pharmacological activities of tetrazoles in the new millennium. Int J Pharm Tech Res 3: 1557-1566. 